\title{
MAGNIFIED CURVES ON A FLAT TORUS, DETERMINATION OF ALMOST PERIODIC FUNCTIONS, AND THE RIEMANN-LEBESGUE LEMMA
}

\author{
ROBERT S. STRICHARTZ
}

(Communicated by J. Marshall Ash)

\begin{abstract}
Simple arguments, based on the Riemann-Lebesgue Lemma, are given to show that for a large class of curves $\gamma$ in $\mathbf{R}^{n}$, any almost periodic function is determined by its restriction to large dilates of $\gamma$. Specializing to periodic functions, this means that magnified images of $\gamma$ on a flat torus tend to uniformly dense scribbles.
\end{abstract}

\section{INTROCUTION}

Suppose you live on a flat torus $\left(\mathbf{R}^{n} / \Gamma\right.$ for a suitable lattice $\left.\Gamma\right)$ and you have a magnifying glass of arbitrarily large power. If you start with a small curve $\gamma$ and subject it to greater and greater magnification, you might expect to see something that resembles a uniformly dense scribble. More precisely, regard $\gamma$ as a subset of $\mathbf{R}^{n}$ and let $\lambda \gamma$ denote the dilates of $\gamma$ by a positive number $\lambda\left(\lambda \gamma=\left\{x \in \mathbf{R}^{n}: x=\lambda y\right.\right.$ for some $\left.\left.y \in \gamma\right\}\right)$. Then $\lambda \gamma \bmod \Gamma$ is the projection of $\lambda \gamma$ onto the torus $\mathbf{R}^{n} / \Gamma$, and we are interested in the behaviour of this projection as $\lambda \rightarrow \infty$. One way to obtain this information is to ask whether a function on the torus is determined more and more precisely by its restriction to $\lambda \gamma \bmod \Gamma$ as $\lambda \rightarrow \infty$.

Functions on a flat torus lift to periodic functions on $\mathbf{R}^{n}$. More generally, consider an almost periodic function $f(x)$ on $\mathbf{R}^{n}$. If $\mu$ is a finite positive measure supported on $\gamma$, then you would like to have

$$
\lim _{\lambda \rightarrow \infty} \int f(\lambda x) d \mu(x)=c M f
$$

where $c=\|\mu\|=\mu\left(\mathbf{R}^{n}\right)$ and $M f$ denotes the Bohr mean of $f$. (Recall that $M f$ is defined to be $\lim _{r \rightarrow \infty}\left|B_{r}\right|^{-1} \int_{B_{r}} f(x) d x$, where $d x$ denotes Lebesgue measure, $B_{r}$ denotes the ball of radius $r$ about the origin, and $\left|B_{r}\right|$ denotes its Lebesgue measure. Notice that this is equivalent to saying

$$
M f=\lim _{\lambda \rightarrow \infty} \int f(\lambda x) d \mu_{1}(x)
$$

Received by the editors July 1, 1988 and, in revised form, May 2, 1989.

1980 Mathematics Subject Classification (1985 Revision). Primary 42A75, 42B10.

Research supported in part by the National Science Foundation, grant DMS-8600245. 
where $\mu_{1}$ denotes normalized Lebesgue measure on the unit ball.) By applying this identity to $e^{i x \cdot \xi} f(x)$ in place of $f$ you could obtain the Fourier series of $f$. Hence in principle you could recover any desired information about $f$.

Clearly not every curve $\gamma$ or every measure $\mu$ will do the trick, so we should ask what conditions should be imposed on $\gamma$ and $\mu$ in order to justify all the above idle speculation. In $\left[R_{1}\right]$, Randol discusses this problem and also gives estimates for the rate of convergence. Here we will not consider rates of convergence, so we can obtain results for more general curves and measures. Our results can be stated entirely in terms of the measure $\mu$. Let us say that $\widehat{\mu}$ (the Fourier transform of $\mu$ ) decays on rays if for every unit vector $u$ we have $\lim _{r \rightarrow \infty} \widehat{\mu}(r u)=0$. Note that we do not require any uniformity in $u$. Thus if $\widehat{\mu}$ vanishes at infinity it decays on rays, but not necessarily conversely.

Lemma 1. Let $\mu$ be a probability measure on $\mathbf{R}^{n}$. The following are equivalent:

(i) $\widehat{\mu}$ decays on rays;

(ii) for any uniformly almost periodic function $f$ on $\mathbf{R}^{n}$,

$$
\lim _{\lambda \rightarrow \infty} \int f(\lambda x) d \mu(x)=M f
$$

(iii) for every flat torus $T=\mathbf{R}^{n} / \Gamma$, the images of the dilates of $\mu$ on $T$ converge to normalized Lebesgue measure on $T$ in the weak topology, i.e., $\lim _{\lambda \rightarrow \infty} \int f(\lambda x) d \mu(x)=c \int_{T} f(x) d x$ for every continuous function fon $T$, where $c^{-1}=\int_{T} d x$.

Proof. Assume (i). Since every uniformly almost periodic function is the uniform limit of trigonometric polynomials ([B]), it suffices to prove (ii) for a trigonometric polynomial, and by linearity for $f(x)=e^{i x \cdot \xi}$. Recall that $M\left(e^{i x \cdot \xi}\right)=0$ if $\xi \neq 0$ and $M(1)=1$. Clearly we have the correct limit for $f \equiv 1$, so it suffices to show

$$
\lim _{i \rightarrow \infty} \int e^{i \lambda x \cdot \xi} d \mu(x)=0 \quad \text { for } \xi \neq 0 .
$$

But this is equivalent to (i). Also (iii) is just a special case of (ii).

Conversely, given (iii), choose $\Gamma$ so that $f(x)=e^{i x \cdot u}$ is periodic with respect to $\Gamma$. Then (iii) gives

$$
\lim _{\lambda \rightarrow \infty} \int e^{i \lambda x \cdot u} d \mu(x)=0
$$

which is (i).

\section{DECAY ON RAYS}

There are many theorems concerning the decay of Fourier transforms of measures (see [S-W] for a few). Most of these theorems establish rates of decay, which for our purpose is irrelevant. Since we only need decay on rays, we can use a very simple tool, the Riemann-Lebesgue Lemma. We do not apply it directly to $\mu$, since if $\mu$ were absolutely continuous our results would 
be banal; but rather we apply it to certain projections of $\mu$. If $u$ is a unit vector define the projection $P_{u} \mu$ of $\mu$ onto the line determined by $u$ to be the measure on $\mathbf{R}$ given by $P_{u} \mu(A)=\mu\left(\left\{A u+u^{\perp}\right\}\right)$ for $A \subseteq \mathbf{R}$ a Borel set, where $A u+u^{\perp}=\left\{x \in \mathbf{R}^{n}: x=a u+v\right.$ where $a \in A$ and $\left.u \cdot v=0\right\}$.

Lemma 2. Suppose $P_{u} \mu$ is absolutely continuous with respect to Lebesgue measure on the line for every unit vector $u$. Then $\hat{\mu}$ decays on rays.

Proof. An easy computation shows that $\widehat{\mu}(\lambda u)$ is the one-dimensional Fourier transform of $P_{u} \mu$. Since $P_{u} \mu$ is clearly a probability measure, the result follows from the one-dimensional Riemann-Lebesgue Lemma.

A remarkable feature of this lemma is that if $\mu$ satisfies its hypotheses then so does the restriction of $\mu$ to any subset of positive measure (multiplied by a suitable constant to obtain a probability measure). Thus, for example, once we have observed that Lebesgue measure on a circle in $\mathbf{R}^{2}$ satisfies the hypotheses then so does Lebesgue measure on an arc of circle, or more generally on any subset of positive measure. This allows us to conclude that a magnified circle on a flat 2-torus scribbles uniformly densely in all directions.

Theorem 1. Let $E \subseteq \mathbf{R}^{n}$ be a Borel set that supports a probability measure $\mu$ such that $P_{u} \mu$ is absolutely continuous for every unit vector $u$. Then

(a) for every torus $T=\mathbf{R}^{n} / \Gamma$ and every $\varepsilon>0$ there exists $\lambda_{0}$ such that $\lambda \geq \lambda_{0}$ implies that $\lambda E$ comes within $\varepsilon$ of every point of $T$ ( for every $x \in \mathbf{R}^{n}$ there exist $y \in E$ and $z \in \Gamma$ such that $|x-z-\lambda y| \leq \varepsilon)$;

(b) if $f_{1}$ and $f_{2}$ are uniformly almost periodic functions and $f_{1}=f_{2}$ on $\lambda_{j}$ E for some sequence $\lambda_{j} \rightarrow \infty$ then $f_{1} \equiv f_{2}$.

Proof. (a) If not, there would be an open ball $B$ of radius $\varepsilon$ such that $\lambda_{j} E$ $\bmod \Gamma$ is disjoint from $B$, for a sequence $\lambda_{j} \rightarrow \infty$ (a priori the center of the ball might vary with $\lambda_{j}$, but since $T$ has finite measure we can select a single ball after all). If we take a positive continuous function $f$ supported in $B$ we have $\int_{T} f(x) d x>0$ but $\int f\left(\lambda_{j} x\right) d \mu(x)=0$ contradicting Lemma 1 (iii).

(b) Since $\left|f_{1}-f_{2}\right|^{2}$ is also uniformly almost periodic, we have

$$
0=\lim _{j \rightarrow \infty} \int\left|f_{1}\left(\lambda_{j} x\right)-f_{2}\left(\lambda_{j} x\right)\right|^{2} d \mu(x)=M\left(\left|f_{1}-f_{2}\right|^{2}\right) .
$$

but $M\left(\left|f_{1}-f_{2}\right|^{2}\right)=0$ implies $f_{1} \equiv f_{2}$ for uniformly almost periodic functions [B].

\section{EXAMPLES}

Consider first the case when $E$ is a $C^{1}$ curve of finite length in the plane, say $\gamma(t)$ parametrized by arc-length, and let $\mu$ be arc-length measure. 
Theorem 2. Assume that $\gamma^{\prime}(t)$ assumes any value at most a finite number of times (or more generally, assume the set $\left\{t: \gamma^{\prime}(t)=u\right\}$ has zero Lebesgue measure for each unit vector $u)$. Then $\widehat{\mu}$ decays on rays.

Proof. Fix a unit vector $u$, and for each fixed $\varepsilon$ write $\mu=\mu_{\varepsilon}+\nu_{\varepsilon}$ where $\mu_{\varepsilon}$ is the restriction of $\mu$ to the portion of the curve where $\left|\gamma^{\prime}(t)-u\right| \geq \varepsilon$. It follows by the change of variable formula for one-dimensional integrals that $P_{u} \mu_{\varepsilon}$ is absolutely continuous with respect to Lebesgue measure, and the result follows since $\left\|\nu_{\varepsilon}\right\| \rightarrow 0$ as $\varepsilon \rightarrow 0$.

Of course the same proof works for surface measure on a $C^{1}$ hypersurface in $\mathbf{R}^{n}$, provided the unit normal to the surface assumes any given value only finitely often (equivalently, the Gauss map is finite-to-one), or more generally on a set of surface measure zero (as is the case, for example, for a cylinder). But there is even an $n$-dimensional version of this result for curves.

Theorem $2^{\prime}$. Let $\gamma(t)$ be a $C^{1}$ curve in $\mathbf{R}^{n}$ of finite length parametrized by arc-length, and let $\mu$ denote arc-length measure along $\gamma(t)$. Suppose for every unit vector $u,\left\{t: \gamma^{\prime}(t) \cdot u=0\right\}$ has Lebesgue measure zero. Then $\widehat{\mu}$ decays on rays.

Proof. Fix $u$, and for each $\varepsilon>0$ write $\mu=\mu_{\varepsilon}+\nu_{\varepsilon}$, where $\mu_{\varepsilon}$ is the restriction of $\mu$ to the part of the curve where $\left|\gamma^{\prime}(t) \cdot u\right| \geq \varepsilon$. The rest of the proof is as before.

In particular, the result applies to any real analytic curve that does not lie in any affine hyperspace. For in that case $\left\{t: \gamma^{\prime}(t) \cdot u=0\right\}$ is defined by an analytic equation, so it is either discrete or the whole line; but we can rule out the latter case because the curve does not lie in any affine hyperspace.

\section{Questions}

Take for $\gamma$ the unit circle in the plane, or more generally an arc of the circle. Our result says that the equations

$$
\begin{aligned}
& r \cos \theta \approx x+j, \\
& r \sin \theta \approx y+k,
\end{aligned}
$$

can be solved for $\theta$ (in fact for $\theta$ in a prescribed interval), where $x$ and $y$ are given, $j$ and $k$ denote integers, and $r$ is taken fixed but large enough, depending on the desired accuracy of the approximation. Is there an explicit formula for doing this? This is not a question of the existence of an algorithm, since trial and error for a sufficiently large set of values for $\theta$ will produce a solution.

A more troubling question concerns the determination of an almost periodic function. Say we know the restriction of an almost periodic function $f$ of $\mathbf{R}^{2}$ to all circles about the origin of integer radius. If we know a priori that the Fourier series of $f$ has the form $\sum a_{j} e^{i x \cdot \xi_{j}}$ for a given sequence $\xi_{1}, \xi_{2}, \cdots$ of 
frequencies (vectors in $\mathbf{R}^{2}$ ) and unknown coefficients $a_{j}$, then we can determine the coefficients by the formula

$$
a_{j}=\lim _{k \rightarrow \infty} \frac{1}{2 \pi} \int_{0}^{2 \pi} f(k \cos \theta, k \sin \theta) e^{-i k(\cos \theta, \sin \theta) \cdot \xi_{j}} d \theta .
$$

But it we are not given a priori information on the frequencies, they can be chosen from an uncountable set, so our formula seems useless. Is there a better formula?

Finally, we can ask similar questions in the context of non-Euclidean geometry. Specifically, suppose $X=H^{2} / \Gamma$ is a compact hyperbolic Riemann surface, where $H^{2}$ is hyperbolic 2-space, say in the Poincare disc model, and $\Gamma$ is a suitable group of isometries $([\mathrm{F}-\mathrm{K}])$. More generally, we could consider the $n$ dimensional analogue $H^{n} / \Gamma$. Randol $\left(\left[\mathrm{R}_{1}\right],\left[\mathrm{R}_{2}\right]\right)$ shows that the images in $X$ of a sequence of dilated spheres in $H^{n}$ become uniformly dense. Is the same true of dilates of other kinds of sets?

\section{REFERENCES}

[B] A. S. Besicovitch, Almost periodic functions, Dover Publications, New York, 1954.

[F-K] H. M. Farkas and I. Kra, Riemann surfaces, Springer-Verlag, New York, 1980.

$\left[\mathrm{R}_{1}\right.$ ] B. Randol, The behavior under projection of dilating sets in a covering space, Trans. Amer. Math. Soc. 285 (1984), 855-859.

[ $\mathrm{R}_{2}$ ] _ , The Selberg trace formula, Chapter XI in Eigenvalues in Riemannian geometry by $\mathrm{I}$. Chavel, Academic Press, 1984.

[S-W] E. M. Stein and S. Wainger, Problems in harmonic analysis related to curvature, Bull. Amer. Math. Soc. 84 (1978), 1239-1295.

Mathematics Department, Cornell University, Ithaca, New York 14853 